\title{
Transzygomatic extended middle fossa approach for upper petroclival skull base lesions
}

\author{
Jin-Cheng Zhao, M.D., and James K. Liu, M.D. \\ Department of Neurological Surgery, Northwestern University Feinberg School of Medicine, Evanston \\ Hospital, NorthShore University HealthSystem, Evanston, Illinois
}

\begin{abstract}
Central skull base lesions in the upper retroclival and petroclival regions can be challenging to access because of their location anterior to the brainstem. Several transpetrosal approaches have been developed to access the petroclival junction, including anterior petrosal (anterior petrosectomy), posterior petrosal (retrolabyrinthine, translabyrinthine, transcochlear), and combined petrosal approaches. The anterior petrosal approach is best suited for upper petroclival lesions located anterior and superior to the internal auditory canal and superior to the inferior petrosal sinus. This approach provides direct access to the anteromedial cerebellopontine angle, petrous apex, Meckel cave, and ventrolateral brainstem between the trigeminal root and the facial nerve. The authors describe their modification of an anterior petrosal approach, the so-called transzygomatic extended middle fossa approach, which incorporates a zygomatic osteotomy, anterior mobilization of the V3, and extensive middle fossa drilling. This exposure provides a wider surgical corridor for direct view of the clivus and ventral brainstem. (DOI: 10.3171/FOC .2008.25.12.E5)
\end{abstract}

\section{KeY WoRdS - anterior petrosectomy - central skull base lesion • extended middle fossa approach • skull base surgery}

$\mathrm{T}$ He middle fossa approach and its various extended modifications have become useful for accessing vascular and neoplastic lesions of the petrous apex, upper petroclival region, petrous ICA, and IAC. In the 1960s, House ${ }^{5,6}$ introduced the middle fossa approach for exposure of the IAC to treat intracanalicular acoustic neuromas with the goal of hearing preservation. The approach was also used for facial nerve exploration and vestibular nerve sectioning. Using the middle fossa corridor, Kawase and colleagues ${ }^{7,8}$ further expanded its indications to include an extradural anterior petrosectomy with subsequent opening of the middle fossa dura mater and division of the SPS and the tentorium to access lower lying basilar artery aneurysms and petroclival meningiomas. This approach has the advantage of providing direct access to the petrous apex, posterior cavernous sinus, upper petroclival region, and anteromedial cerebellopontine angle, while minimizing temporal lobe retraction and preserving the auditory and vestibular apparatus. $^{1}$

Some technical modifications have been reported to increase the surgical corridor. MacDonald et al. ${ }^{10}$ described performing a petrous apex resection combined

Abbreviations used in this paper: IAC = internal auditory canal; ICA = internal carotid artery; GSPN = greater superficial petrosal nerve; SPS = superior petrosal sinus; $\mathrm{TMJ}=$ temporomandibular joint. with an anterior subtemporal approach that provided a wider exposure to view the upper basilar artery and clivus and the ventral surface of the mesencephalon and upper pons. Through the anteromedial removal of the petrosal bone, the midbasilar and upper clival basilar artery region above the internal auditory meatus can be accessed. Liu et al. ${ }^{9}$ described the addition of a zygomatic osteotomy and more extensive removal of middle fossa bone (removal of the Glasscock and Kawase triangles) to increase exposure of the petrous ICA for cerebrovascular bypass procedures. Cho and Al-Mefty ${ }^{2}$ described combining the anterior petrosectomy with a retrolabyrinthine petrosectomy (combined petrosal approach), which provided multidirectional access to petroclival meningiomas while preserving the hearing apparatus and avoiding transposition of the facial nerve.

We describe a transzygomatic extended middle fossa approach in which the surgical corridor is increased and exposure of the petrous apex and upper petroclival region of the central skull base is maximized. This approach incorporates a temporal or frontotemporal craniotomy, zygomatic osteotomy, extradural exposure of the middle fossa, anterior mobilization of the V3, and extensive drilling of the middle fossa skull base. This exposure provides direct access to the posterior cavernous sinus, Meckel cave, petrous apex, upper petroclival region, anteromedial cerebellopontine angle, basilar trunk, and anterolateral brainstem from the trigeminal root to the porus acusticus. This approach can be applied to petroclival 

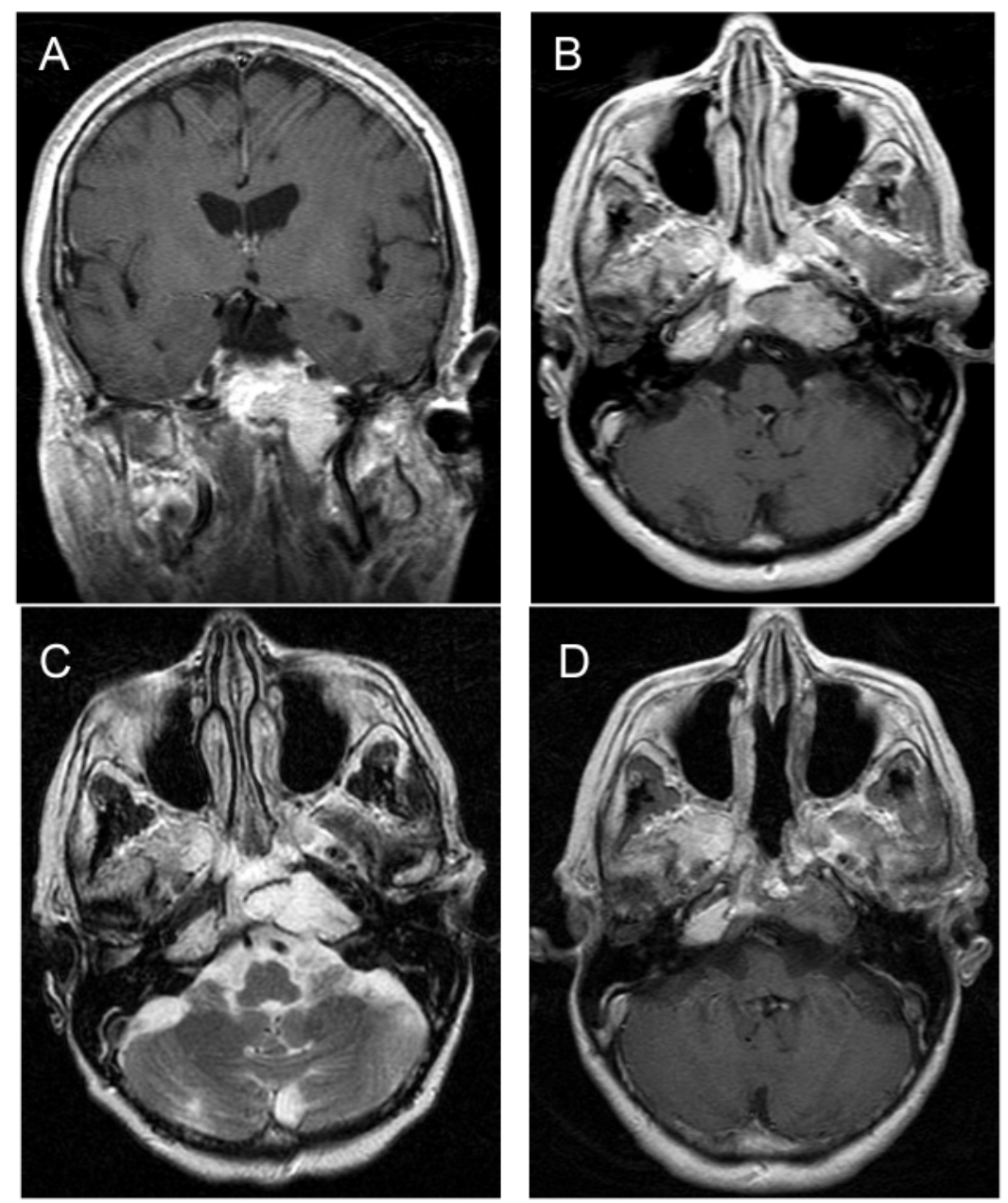

FIG. 1. A-C: Preoperative T1-weighted Gd-enhanced coronal (A) and axial (B) and T2-weighted axial (C) MR images obtained in a 55-year-old woman demonstrating a hyperintense enhancing lesion in the petrous apex and upper petroclival region. The lesion is situated medial to the petrous ICA and anterior to the IAC. An extended middle fossa approach was performed to resect the tumor, which was a pathologically proven low-grade chondrosarcoma.

D: Postoperative Gd-enhanced MR image showing

meningiomas, chordomas, chondrosarcomas, trigeminal schwannomas, cholesterol granulomas, mid-basilar trunk aneurysms, and cavernous malformations of the anterolateral pons (Figs. 1 and 2). Exposure of the petrous ICA for revascularization options can also be performed. ${ }^{9}$

\section{Operative Description}

\section{Frontotemporal Craniotomy and Zygomatic Osteotomy}

Prior to positioning the patient, an intraoperative lumbar drain is placed to facilitate brain relaxation and minimize temporal lobe retraction. The patient is positioned supine with the ipsilateral shoulder slightly elevated with a shoulder roll. The head is rotated $45^{\circ}$ to the contralateral side in a 3-pin head holder with the malar eminence positioned at the highest point. A frontotemporal curvilinear skin incision is made starting at the level of the zy- goma and $1 \mathrm{~cm}$ anterior to the tragus. This avoids injury to the frontalis branch of the facial nerve as it crosses over the zygomatic arch. The incision is carried superiorly and then anteriorly, staying behind the hairline. The scalp is elevated in a 2-layer fashion. Initially, the galeal layer is elevated while the pericranium is left on the skull. The pericranium is then elevated as a separate layer, which remains attached to its vascularized pedicle for reconstruction at the time of closure, should it be necessary. Interfascial dissection of the temporalis muscle is performed to expose the orbit and zygoma while protecting the frontotemporal branch of the facial nerve. , $^{3}$

Two cuts are made in the zygomatic arch (Fig. 3). The first cut is created through the root of the zygoma, just anterior to the TMJ and the glenoid fossa. This cut should begin at the superoposterior aspect of the root of the zygoma and move obliquely toward the anteroinferior point. A cut made in this direction minimizes overhang of bone from the root of the zygoma and also allows for 

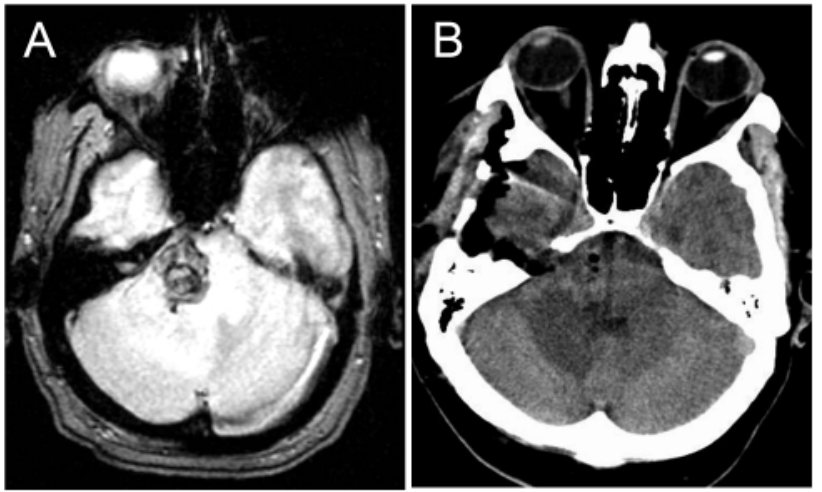

FIG. 2. A: Gradient echo MR imaging demonstrating a hemorrhagic lesion of the anterolateral pons in a 46 year old male consistent with a brain stem cavernous malformation. The lesion appeared to come to the surface at the anterolateral aspect of the pons between the trigeminal and facial nerves. An extended middle fossa approach was used to perform gross-total resection of the lesion. The lesion was pathologically determined to be a renal cell metastasis. B: Postoperative CT scan showing gross total resection.

cosmetic reattachment. The second cut, which is parallel to the lateral orbital rim, begins at the frontozygomatic suture and leaves as little bone overhanging the frontozygomatic recess as possible. The zygomatic arch can be removed by freeing attachments from the masseter muscle. Alternatively, the arch can be displaced inferiorly and attachments to the masseter muscle can be preserved. If additional anterolateral skull base and orbitofrontal exposures are needed, a full orbitozygomatic osteotomy, as described by Zabramski et al. ${ }^{12}$ can also be performed using this exposure.

The temporalis muscle is then incised horizontally along the superior temporal line and vertically along the plane of the preauricular incision, leaving a $1.5-\mathrm{cm}$ myofascial cuff along the superior temporal line for reattachment at the time of closure. The temporalis muscle is retracted inferiorly with blunt fish hooks and rubber bands to expose the infratemporal fossa (Fig. 4).

A standard frontotemporal craniotomy is performed. Any remaining squamous temporal bone is removed so that the edge of the craniotomy becomes flush with the middle fossa floor. The lesser wing of the sphenoid is removed extradurally using rongeurs and a high-speed drill to provide a flat trajectory to the skull base.

\section{Extradural Exposure of the Middle Cranial Fossa and Posterior Cavernous Sinus}

The osseous floor of the middle fossa and the posterior cavernous sinus are then exposed by gently elevating the temporal lobe in an extradural fashion (Fig. 5). Dissection is carried out in a posteroanterior direction. Care is taken to avoid a traction injury to the facial nerve, which is caused by excessive stretching of the GSPN as it is separated from the temporal lobe dura. The arcuate eminence is found along the posterior petrous ridge. Often there are 2 osseous humps in the middle fossa, which look like a double ridge. The more medial ridge of bone usually represents the arcuate eminence. Elevation of the

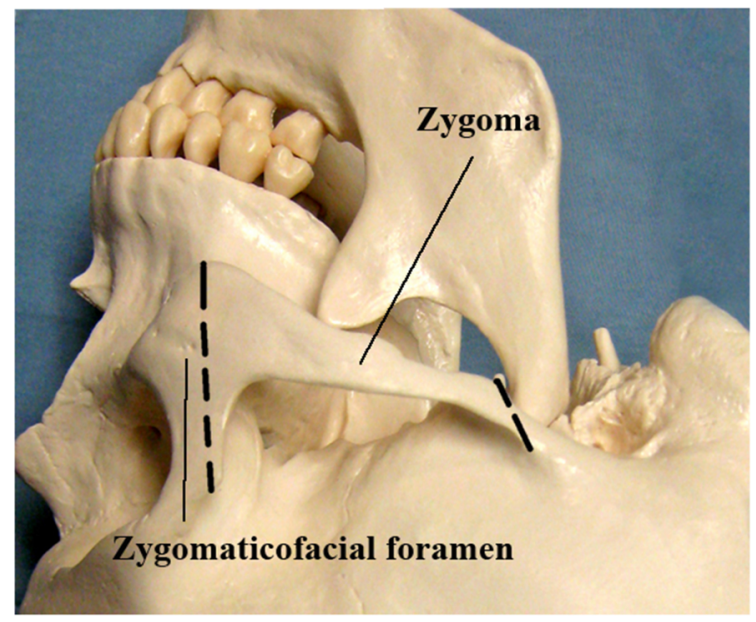

FIG. 3. Photograph of a skull model demonstrating where the bone cuts are made for the zygomatic osteotomy.

dura is continued anteromedially to expose the region of the geniculate ganglion and the GSPN, which lies in the major petrosal groove and is covered by a layer of connective tissue. The middle meningeal artery is coagulated and divided where it enters the foramen spinosum. This allows further release of the dura propria, which is continuous with the temporal lobe dura. The dura mater propria can now be lifted from the lateral wall of the cavernous sinus, and the mandibular division of the trigeminal nerve (V3) exposed as it exits the foramen ovale. Elevation of the dura is carried medially to the extent of the medial petrous ridge. The V3 is retracted anteriorly by using a

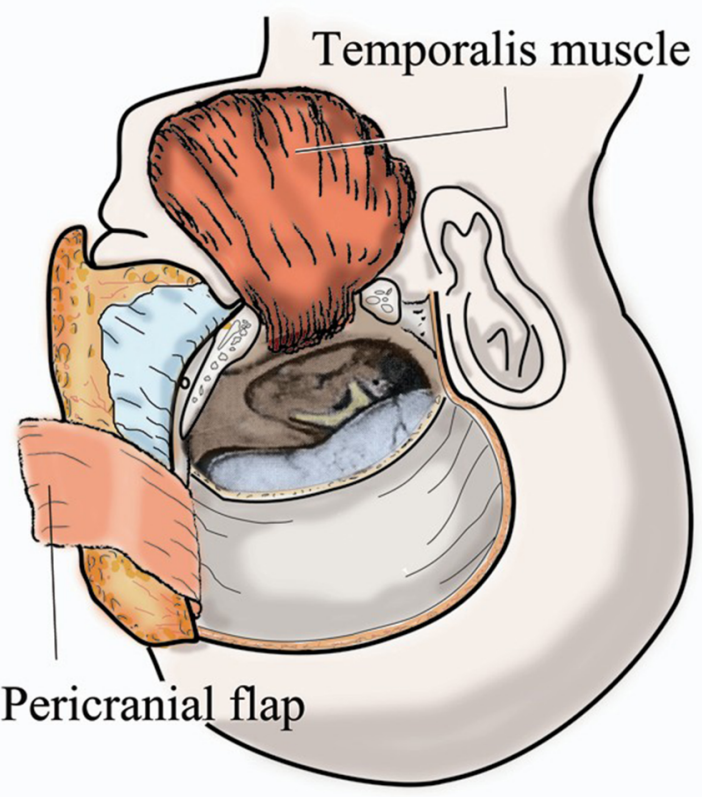

FIG. 4. Illustration showing the exposure after the frontotemporal craniotomy and zygomatic osteotomy have been performed. The temporal lobe dura is elevated extradurally to expose the floor of the middle fossa. 

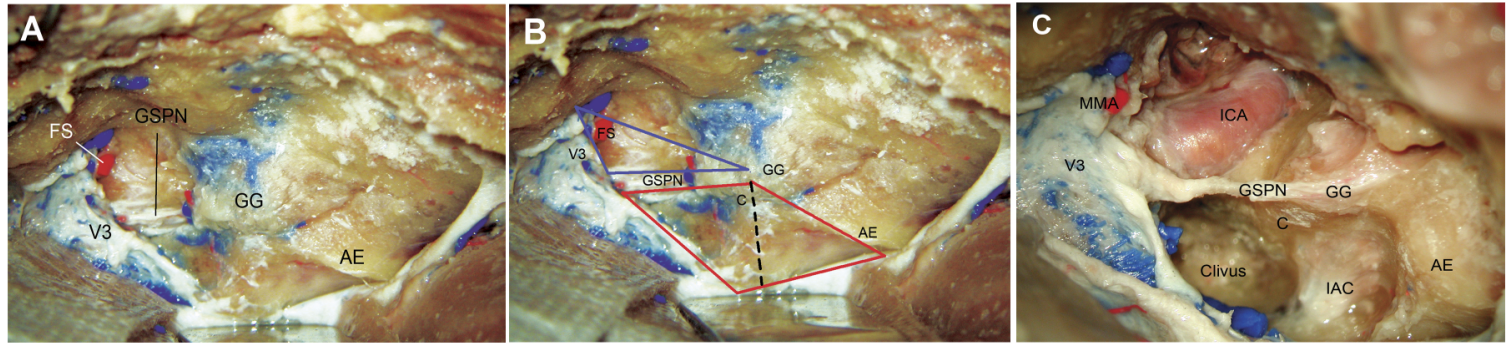

FIG. 5. A: Photograph of a cadaveric dissection via a right-sided transzygomatic extended middle fossa approach; the anatomy of the middle fossa is demonstrated. The dura of the temporal lobe has been elevated extradurally in a posteroanterior direction to expose the floor of the middle fossa and the GSPN. The middle meningeal artery (MMA) at the foramen spinosum (FS) has been divided to allow further release of the temporal lobe dura to expose the posterior cavernous sinus and the V3. B: The middle fossa rhomboid (red) is bordered by the V3 anteriorly, the GSPN laterally, the arcuate eminence (AE) posteriorly, and the petrous ridge medially. The horizontal segment of the petrous ICA courses parallel to and deep with respect to the GSPN. The IAC (black dotted line) lies approximately in the plane that bisects the angle between the GSPN and the AE. The cochlea (C) is situated anteromedial and inferior to the geniculate ganglion (GG). The Glasscock triangle (blue) is surrounded by the posterior rim of the foramen ovale, the foramen spinosum, the posterior border of the V3, and the cochlear apex. C: Extensive removal of bone of the middle fossa rhomboid and the Glasscock triangle provides exposure of the petrous ICA, clivus, and IAC.

self-retaining retractor blade to provide more exposure of the petrous apex.

Orientation of the middle fossa anatomy can be achieved by identifying the anatomical landmarks of the middle fossa rhomboid structure as described by Day et al. ${ }^{4}$ The 4 sides of the rhomboid are bordered by the V3 anteriorly, the GSPN laterally, the arcuate eminence posteriorly, and the petrous ridge medially (Fig. 5). The middle fossa rhomboid serves as a useful landmark to identify the petrous ICA and to avoid important neurootological structures during drilling of petrous bone. The horizontal segment of the petrous ICA may be visible through a bone dehiscence in the floor of the middle fossa in some cases; the segment courses parallel to and deep with respect to the GSPN. The IAC lies approximately in the plane that bisects the angle between the GSPN and the arcuate eminence. The cochlea is situated anteromedial and inferior to the geniculate ganglion. The arcuate eminence serves as a bony landmark of the superior semicircular canal.

\section{Extradural Drilling of Middle Fossa Rhomboid}

An anterior petrosectomy is performed by removing the bone of the middle fossa rhomboid with care to preserve the neurovascular and neurootological structures. This is done in a stepwise fashion using a high-speed diamond bur and copious irrigation. We initially identify the IAC, which lies in the bisection of the angle formed by the GSPN and the arcuate eminence. Drilling is performed mediolaterally, beginning at the medial petrous edge, to unroof the medial two-thirds of the IAC. The dura overlying the medial IAC flares out at the porus acusticus. Bone is removed between the posterior edge of the V3 and the anterior edge of the IAC. Anterior retraction of the V3 allows further anteromedial exposure of the petrous apex to facilitate the petrosectomy (Fig. 6).
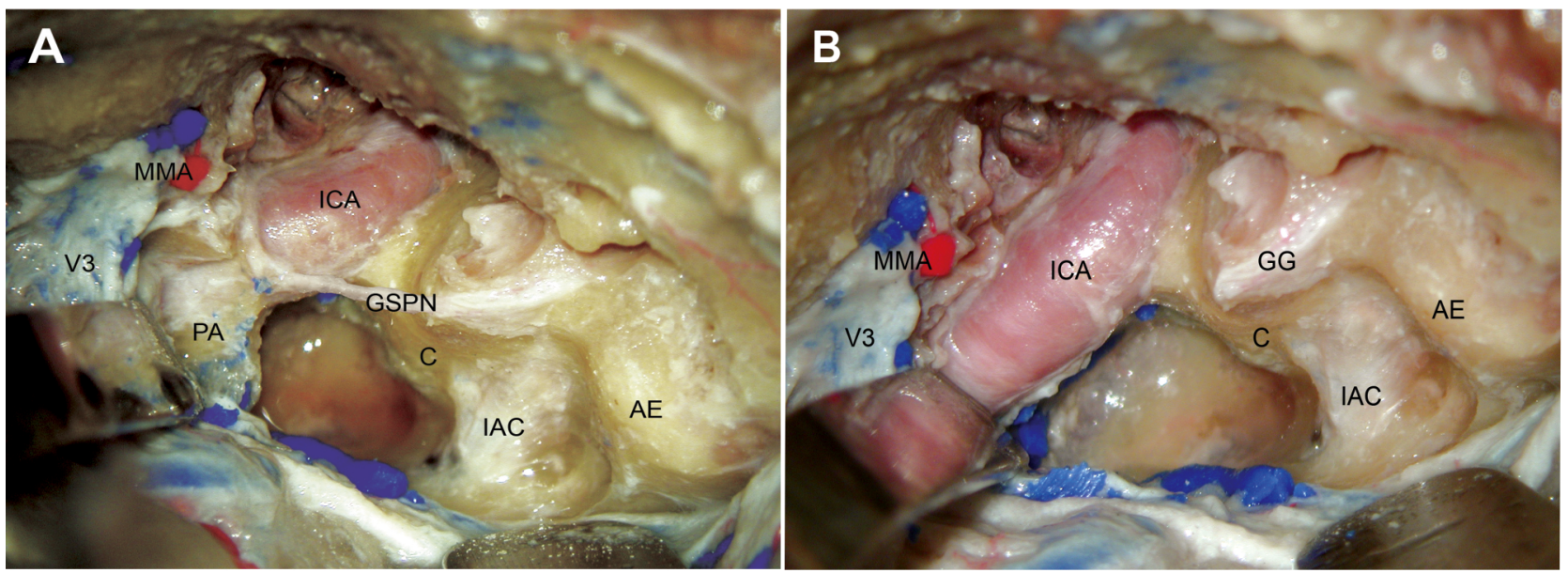

FIG. 6. Photographs of a cadaveric specimen. A: Anterior retraction of the V3 provides additional exposure of the petrous apex (PA) to be removed. B: Further removal of the anterior petrous apex has been performed medial and inferior to the trigeminal nerve down to the depth of the inferior petrosal sinus, providing additional exposure of the ICA. In this photograph, the GSPN has been divided to prevent traction injury to the facial nerve during bone drilling. 

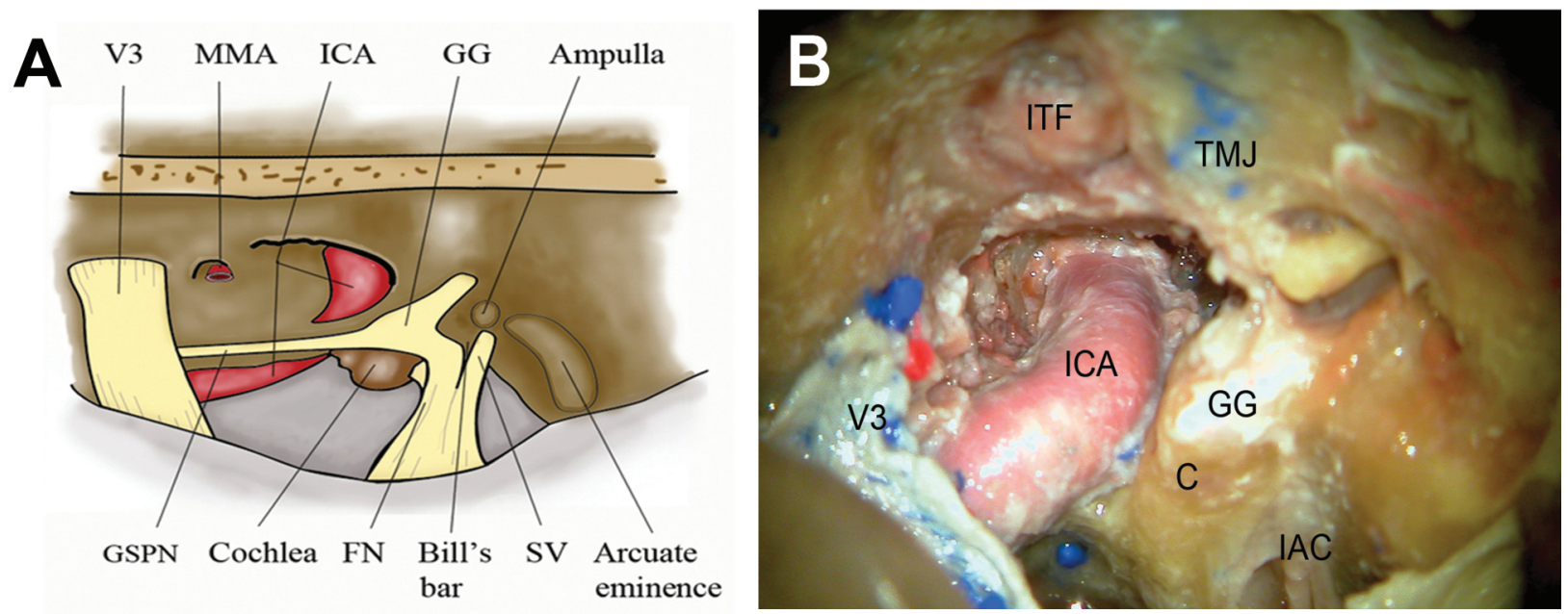

FIG. 7. Artist's illustration $(A)$ and cadaveric dissection photograph $(B)$ demonstrating complete exposure of the petrous ICA including the horizontal segment, genu, and vertical segment. The anterior and lateral aspects of the vertical segment of the petrous ICA have been unroofed completely down to the carotid canal entrance at the cranial base. The floor of the middle fossa lateral to the horizontal segment and anterior to the vertical segment has been removed to reveal the infratemporal fossa (ITF).

Bone is removed inferiorly and medially until the inferior petrosal sinus is visualized. The lateral border of the petrosectomy is the horizontal segment of the petrous ICA. The cochlea, which is located anteromedial and inferior to the geniculate ganglion, must be preserved if hearing conservation is the goal. Last, the bone between the IAC and the arcuate eminence is removed.

If exposure of the petrous ICA is desired, the bone overlying the Glasscock triangle can be removed. The Glasscock triangle is bordered by the posterior rim of the foramen ovale, the foramen spinosum, the posterior border of the V3, and the cochlear apex. We prefer to transect the GSPN here to prevent traction injury tothe facial nerve during skeletonization of the horizontal segment of the petrous ICA. Additional exposure of the distal horizontal segment can be achieved with anterior retraction of the V3. Complete skeletonization of the entire petrous ICA (horizontal segment to the genu and to the vertical segment) can be achieved through this exposure, if needed (Fig. 7). The bone lateral to the horizontal petrous ICA and anterior to the vertical segment can be removed down to the infratemporal fossa to provide a wider angle of exposure and an inferosuperior trajectory to the petrous ICA. Care is taken to avoid injury to the TMJ, which is posterolateral to the vertical segment of the petrous ICA. The eustachian tube is situated lateral to the horizontal petrous ICA and should be preserved if possible. However, if the eustachian tube is inadvertently violated, obliteration of the tube is essential to prevent a CSF fistula.

\section{Intradural Exposure of the Posterior Fossa}

For access to intradural lesions, the dura is opened inferiorly along the floor of the middle fossa. The tentorium cerebelli is then divided sharply posterior to the trochlear nerve as it enters the tentorial edge. The incision extends laterally to the SPS, which is ligated with titanium clips and divided sharply. Another incision extends perpendicularly from the middle fossa incision across the SPS and inferiorly into the posterior fossa dura. ${ }^{11}$ This provides intradural access to the anterolateral brainstem from the level of the sellar floor to the IAC. This exposure is useful for access to the basilar trunk and also for intrinsic lesions, such as cavernous malformations, of the brainstem between the trigeminal root and facial nerve (Figs. 2 and 8).

\section{Closure}

The horizontal dural incision along the temporal base is reapproximated as well as possible. Water-tight closure is difficult to achieve in cases in which a transtentorial incision into the middle fossa rhomboid was performed. A dural allograft placed in an onlay fashion can be used in conjunction with dural sealants. The petrous apex defect and any pneumatized cells are obliterated with an autologous fat graft. The fat graft is also used to bolster any dural defects. Care is taken not to overpack the fat graft, as this may cause temporal lobe compression and mass effect. If necessary, a vascularized pedicled pericranial flap or temporalis muscle flap can be swung into the temporal base for reconstruction. The bone flap is secured with titanium screws and plates. If there is a significant temporal squama or pterional defect, the senior author (J.K.L.) prefers to perform a cranioplasty using the Medpor Titan implant (Porex Surgical), which is a sheet of titanium mesh embedded between 2 sheets of porous polyethylene. The implant is thin and malleable and can be easily cut to fashion the skull defect. The implant is readily secured with several titanium screws to the adjacent bone flap. Lumbar catheter drainage at $10 \mathrm{ml}$ per hour is used for 3 to 4 days postoperatively to prevent CSF leakage if an intradural exposure was performed. 

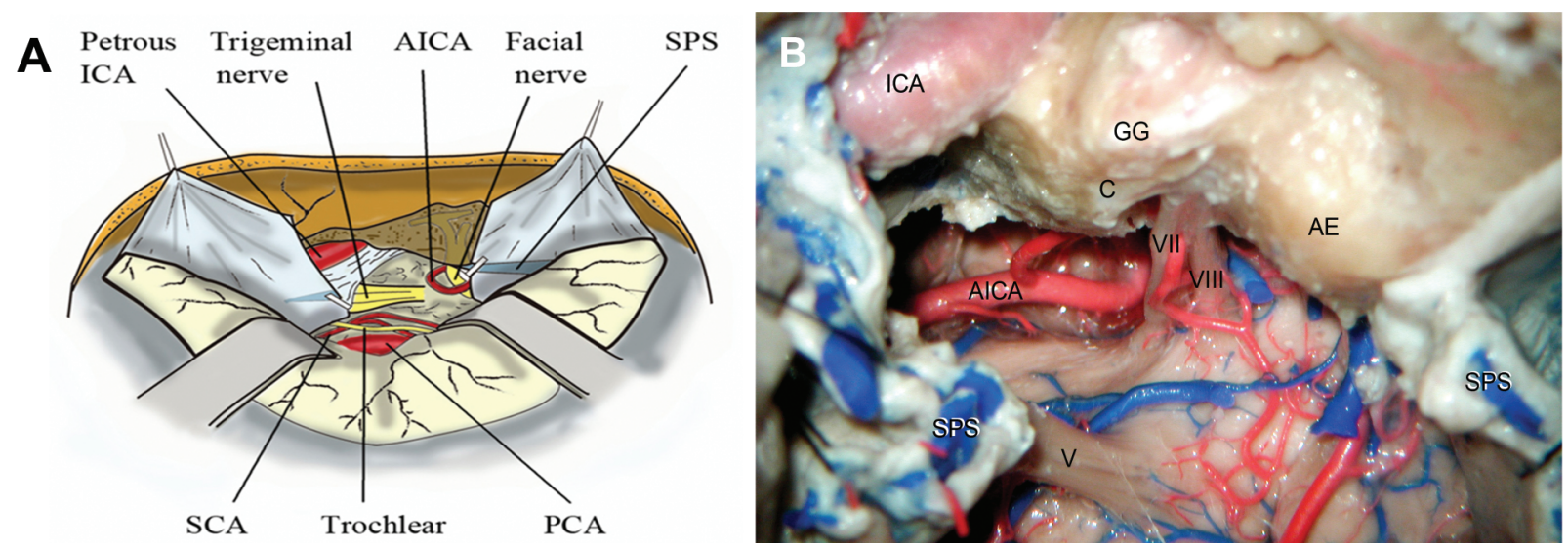

FIG. 8. Illustration (A) and cadaveric dissection photograph (B) demonstrating the intradural exposure of the anterolateral brainstem. The dura is incised along the base of the temporal lobe and the tentorium is incised posterior to the entrance of the trochlear nerve into the tentorium. The incision is carried into the Kawase triangle and into the posterior fossa dura where the SPS is ligated and divided. Excellent exposure of the anterolateral brainstem can be achieved between the root of CN V and CN $\mathrm{VII}$ and VIII. The anterior inferior cerebellar artery (AICA) can be visualized in this dissection. PCA = posterior cerebral artery; SCA = superior cerebellar artery.

\section{Conclusions}

The transzygomatic extended middle fossa approach provides a wide surgical corridor for accessing the posterior cavernous sinus, petrous apex, and upper petroclival region. Excellent visualization of the anteromedial cerebellopontine angle can be achieved for the surgical treatment of lesions between the trigeminal and facial nerves and lesions located inferiorly on the basilar trunk. Knowledge of the middle fossa rhomboid structure is useful for anatomical orientation and avoiding injury to neurovascular and neurootological structures. Surgical rehearsal in a skull base microsurgical laboratory is highly recommended.

\section{References}

1. Aziz KM, van Loveren HR, Tew JM Jr, Chicoine MR: The Kawase approach to retrosellar and upper clival basilar aneurysms. Neurosurgery 44:1225-1226, 1999

2. Cho CW, Al-Mefty O: Combined petrosal approach to petroclival meningiomas. Neurosurgery 51:708-718, 2002

3. Coscarella E, Vishteh AG, Spetzler RF, Seoane E, Zabramski JM: Subfascial and submuscular methods of temporal muscle dissection and their relationship to the frontal branch of the facial nerve. Technical note. J Neurosurg 92:877-880, 2000

4. Day JD, Fukushima T, Giannotta SL: Microanatomical study of the extradural middle fossa approach to the petroclival and posterior cavernous sinus region: description of the rhomboid construct. Neurosurgery 34:1009-1016, 1994

5. House WF: Middle cranial fossa approach to the petrous pyramid. Report Of 50 Cases. Arch Otolaryngol 78:460-469, 1963

6. House WF: Surgical exposure of the internal auditory canal and its contents through the middle, cranial fossa. Laryngoscope 71:1363-1385, 1961

7. Kawase T, Shiobara R, Toya S: Anterior transpetrosal-transtentorial approach for sphenopetroclival meningiomas: surgical method and results in 10 patients. Neurosurgery 28: 869-876, 1991

8. Kawase T, Toya S, Shiobara R, Mine T: Transpetrosal ap- proach for aneurysms of the lower basilar artery. $\mathbf{J}$ Neurosurg 63:857-861, 1985

9. Liu JK, Fukushima T, Sameshima T, Al-Mefty O, Couldwell WT: Increasing exposure of the petrous internal carotid artery for revascularization using the transzygomatic extended middle fossa approach: a cadaveric morphometric study. Neurosurgery 59:309-319, 2006

10. MacDonald JD, Antonelli P, Day AL: The anterior subtemporal, medial transpetrosal approach to the upper basilar artery and ponto-mesencephalic junction. Neurosurgery 43:84-89, 1998

11. Miller CG, van Loveren HR, Keller JT, Pensak M, el-Kalliny M, Tew JM Jr: Transpetrosal approach: surgical anatomy and technique. Neurosurgery 33:461-469, 1993

12. Zabramski JM, Kiris T, Sankhla SK, Caboil J, Spetzler RF: Orbitozygomatic craniotomy. Technical note. J Neurosurg 89:336-341, 1998

Manuscript submitted August 15, 2008.

Accepted October 13, 2008.

Address correspondence to: James K. Liu, M.D., Department of Neurological Surgery, Northwestern University Feinberg School of Medicine, Evanston Hospital, 2650 Ridge Avenue, Evanston, Illinois 60201. email: jliu@enh.org

\section{Comment}

The report describes a brave version of the transpetrous approach. The transpetrous approach has been repeatedly described by different authors. In our anatomical studies of the parasellar compartment and adjacent regions, originally published in 1989, the safest trajectory from the middle cranial fossa to the posterior cranial fossa is described as traversing the petrous bone. The inferomedial triangle and the inferolateral triangle, with its tentorial part and with its osseous portion, were discussed in detail in that publication. ${ }^{1}$ In a separate publication, ${ }^{2}$ the safest possible resection of the apex of the pyramid behind and underneath $\mathrm{CN} \mathrm{V}$, in front of the IAC, was described in detail. The structures at the borderlines of this corridor were also discussed in detail. By traversing the apex of 


\section{Transzygomatic extended middle fossa approach}

the petrous bone from the middle cranial fossa, one can avoid injury to CN VII and VIII in the IAC and injury to CN VI in the Dorello canal. This corridor exposes the midportion of the clival region widely (enough). In cases in which it is necessary to visualize the more superior part of the clival region and/or the posterior aspect of the posterior clinoid process (PCP), the anterior approach is used in combination with the transpetrous approach. The so-called trans-ACP (anterior clinoid process) and transPCP approach to the posterior fossa, in combination with the transpetrous approach, provides an approach to the upper half of the clivus and includes the exposure of the whole length of the basilar artery, from the junction point of both vertebral arteries, up to the basilar tip and the exposure of the brainstem, accordingly.

The approach presented by the authors does describe the transection of the greater petrosal nerve. Most probably, this is not the best idea. In our practice, we always perform resection of the apex of the pyramid after visualization of the IAC, and we decide if it is necessary to extend the approach more toward the clivus (medially). In cases of petroclival meningioma in which the tumor is also on the proximal side of $\mathrm{CN} \mathrm{V}$, the dura of the medial aspect of the apex of the pyramid is also cut toward the SPS, which in most cases is already occluded. It is, however, important that the transsection of the SPS be performed on the right side in order to avoid injury to the inflow of the petrosal vein into the SPS, the consequences of which are well known. Next, the dural membrane around the $\mathrm{CN} \mathrm{V}$ is cut, and the segment of CN V in transition from the posterior cranial fossa to the Meckel cave is well visualized and can be dissected from the tumor and then gently retracted posteriorly. With this maneuver, the so-called posterior area of the Parkinson triangle is maximally enlarged, still without endangering CN IV. Any possible remaining osseous portion of the apex of the pyramid can be drilled off the anterior side of $\mathrm{CN} \mathrm{V}$, and the petroclival ligament can be visualized and the remaining tumor resected. The tentorial edge should be kept intact, since it provides "natural" protection for $\mathrm{CN}$ IV until the end of tumor resection. Resection of the lesion toward and in the Dorello canal is then possible, and CN VI is visualized and dissected from the brainstem to the posterior part of the parasellar space. In cases in which the tumorous lesion is located even more laterally or ventrally, it can be easily followed. As is true of surgery in general, the golden principle is that the approach-actually the extent of the approach-should be tailored to the individual case. There is no need to explore structures that are not on the way.

\section{References}

1. Dolenc VV: Anatomy and Surgery of the Cavernous Sinus. New York: Springer Wien, 1989, pp. 57-86.

2. Dolenc VV, Rogers L: Microsurgical Anatomy and Surgery of the Central Skull Base. New York: Springer Wien, 2003, pp 26-34, 51-72, 185-191, 212-221.

Vinko Dolenc, M.D., Ph.D. International Institute for Neurosurgery and Neuroresearch Ljubljana, Slovenia 